\title{
Influencia de la composición y tratamiento térmico sobre el comportamiento a fluencia de aceros ferrítico-martensíticos del tipo 9Cr1MoVNb utilizados en calderas supercríticas
}

\author{
I. GUTIERREZ URRUTIA ${ }^{1}$, S. GUTIERREZ DE SAIZ-SOLABARRIA ${ }^{2}$ y J.M. SA JUAN ${ }^{1}$ \\ ${ }^{1}$ Dpto. Física de la Materia Condensada. Facultad de Ciencias (Campus de Leioa). \\ Universidad del País Vasco (UPV/EHU). Apdo. 644. Bilbao 48080. \\ ${ }^{2}$ Dpto. Ciencia de Materiales e Ing. Metalúrgica. Univ. País Vasco. Apdo. 644. Bilbao 48080 y Dpto. Lab. \\ Unidad I+D. Babcock \& Wilcox Spain S.A. Apdo. 294. Bilbao 48080.
}

\begin{abstract}
Se estudia el comportamiento a fluencia de varios aceros ferrítico-martensíticos de última generación, del tipo 9Cr1MoVNb, que son ampliamente utilizados como tubos presurizados de alta temperatura en calderas supercríticas para centrales térmicas avanzadas de producción de energía eléctrica. El material utilizado fue fabricado en hornos de inducción de alta frecuencia y al vacío, laminado en caliente y posteriormente aplicado diferentes tratamientos térmicos de Normalizado y Revenido. En los ensayos de fluencia se aplicaron diferentes y ctes. $\sigma<\operatorname{Re}$ y temperaturas de 550, 600 y $650^{\circ} \mathrm{C}$. La influencia de la variación de determinados elementos $(\mathrm{C}, \mathrm{N}, \mathrm{V}, \mathrm{Nb}, \mathrm{Ti}, \mathrm{B})$ de su composición química así como observar los valores que toman los parámetros de Larson-Miller, Manson-Haferd y Orr-Sherby-Dorn, son asimismo contemplados.
\end{abstract}

Palabras clave: Aceros tipo 9Cr1MoVNb, composición química, tratamientos térmicos, comportamiento a fluencia.

Influence of composition and heat treatment in the creep behaviour of ferritic-martensitic steels (type 9Cr1MoVNb) used for supercritical boilers

In the present investigation, creep behaviour on various ferritic-martensitic steels (type 9Cr1MoVNb) from last generation were studied. They're amply used as high pressure and temperature tubes in supercritical boilers for advanced thermoelectric plant. The material used was produced with vacuum high frecuency induction furnace hot-rolling followed by normalizing plus and tempering heat treatments. Creep testing carry out with different stress (also constant) lower than yield strength $(\sigma<\mathrm{Re})$ and temperatures at $600 \pm 50^{\circ} \mathrm{C}$. Influence by variation of some chemical elements $(\mathrm{B}, \mathrm{Ti}, \mathrm{V}, \mathrm{Nb}, \mathrm{V}, \mathrm{C},)^{2}$ as well assessment Larson-Miller, Manson-Haferd and Orr-Sherby-Dorn parameters, they're likewise studied.

Key words : Steels type 9Cr1MoVNb, chemistry composition, heat treatment, creeping behaviour.

\section{INTRODUCCION}

Se estudia la influencia de la composición y tratamiento térmico sobre el comportamiento a fluencia de varios aceros ferrítico-martensíticos, de última generación, del tipo 9Cr1MoVNb, desarrollados, en un principio (1), por Oak Ridge National Laboratories (ORNL) conjuntamente con Combustion Engineering en USA, conocidos por ASME/ $\operatorname{ASTM}(2,3,4)$ con las designaciones T91/P91 y en Europa como X10CrMoVNb9.1 (5). Son aceros dúctiles y tenaces que presentan muy buenas propiedades de resistencia a fluencia, soldabilidad y conductividad térmica $(1,6,7,8)$. Son cada vez más usados como tubos de alta temperatura $\left(600 \pm 50^{\circ} \mathrm{C}\right)$ y presión [300 bar $\left.\left(30 \mathrm{~N} / \mathrm{mm}^{2}\right)\right]$ en calderas supercríticas para centrales térmicas avanzadas $(9,10)$.

El rival más directo es el acero inoxidable martensítico X20CrMoV12-1 (12CrMoV) pero éste como consecuencia de su mayor contenido en carbono puede presentar algún problema en su soldabilidad y por ende la tenacidad de la unión soldada se verá disminuida (11).

De los trabajos de Arav-Van Mortel $(12,13)$ y de Bell (1) observamos que para las mismas condiciones de servicio $(\mathrm{T}=$ $\left.600^{\circ} \mathrm{C} ; \mathrm{p}=300 \mathrm{bar}\right)$ los tubos de P91 ( $\left.9 \mathrm{Cr} 1 \mathrm{MoVNb}\right)$ necesitarían un espesor de $49 \mathrm{~mm}$, frente a los $77 \mathrm{~mm}$ del X20 (X20CrMoV12-1) y 115 mm del P22 (21/4Cr1Mo) para un tiempo de operación de 100.000 horas ( $\approx 12$ años).

Las coladas experimentales han sido fabricadas en hornos de inducción de alta frecuencia y al vacío y posteriormente laminadas en caliente.

El presente trabajo es continuación de los presentados en el V Congreso de Propiedades Mecánicas de Sólidos (14) y Tratermat'98 (15), abordando ahora el estudio del comportamiento a fluencia.

\section{PROCEDIMIENTO EXPERIMETAL}

\subsection{Materiales}

Se fabricaron siete coladas experimentales (UPV. 1, 2, 3, 8, 9, 11 y 12) en hornos de inducción de alta frecuencia y al vacío. Se obtuvieron lingotes de $50 \mathrm{Kg}$ que posteriormente se laminaron 
TABLA I. COMPOSICIONES QUÍMICAS DE LAS 7 COLADAS EXPERIMENTALES Y LA COLADA COMERCIAL (UPV-15). (LOS VALORES SUBRAYADOS SON AQUELLOS QUE SE DESVÍAN DE LA COMPOSICIÓN ESPECIFICADA POR ASME/ASTM PARA LA CALIDAD SA/A 213 GR T91)

\begin{tabular}{|c|c|c|c|c|c|c|c|c|c|c|c|c|c|c|c|c|c|}
\hline \multirow{2}{*}{$\begin{array}{c}\text { ACERO } \\
\left(\mathrm{N}^{0}\right)\end{array}$} & \multicolumn{13}{|c|}{ Composición química, \% en masa } & \multirow{2}{*}{$\begin{array}{c}\mathrm{Cr} \\
\text { Equiv. }\end{array}$} & \multirow{2}{*}{$\begin{array}{c}\mathrm{Ni} \\
\text { Equiv. }\end{array}$} & \multicolumn{2}{|c|}{ Fe $\delta$} \\
\hline & c & $\mathrm{Si}$ & Mn & $\mathbf{P}$ & $\mathbf{s}$ & $\mathrm{Cr}$ & Mo & $\mathrm{Ni}$ & $\mathrm{N}$ & $\mathrm{Nb}$ & $\mathrm{Ti}$ & $v$ & Otros & & & $\begin{array}{l}\text { Pred. } \\
\text { (Fig.1) }\end{array}$ & Actuat \\
\hline UPV.I & .092 & .28 & .4 & . & .005 & 8.72 & 1.00 & .10 & .044 & .08 & $<.005$ & .23 & .. & 12.1 & 4.2 & No & 0 \\
\hline UPV.2 & $\underline{.053}$ & .31 & .45 & .008 & .003 & 8.88 & .99 & .10 & .039 & .08 & $<.02$ & .23 & .- & 12.3 & 3.9 & Si & 0 \\
\hline UPV.3 & .031 & .31 & .45 & .008 & .003 & 8.93 & .99 & .10 & .073 & .08 & $<.02$ & .22 & .- & 12.3 & 3.1 & Si & Trazas \\
\hline UPV .8 & .086 & .29 & .44 & .009 & .006 & 8.90 & 1.00 & .10 & .043 & .08 &.. &.$\underline{.50}$ & .- & 13.6 & 4.0 & $\mathrm{Si}$ & Truzas \\
\hline UPV .9 & .090 & .29 & .44 & .008 & .005 & 8.71 & .99 & .11 & .038 & .15 &.. & .23 & .- & 12.2 & 4.0 & No & 0 \\
\hline UPV.II & .094 & .32 & .44 & .002 & .005 & 8.85 & .99 & .10 & .040 & .08 & $<.01$ & .24 & -- & 12.3 & 4.1 & No & 0 \\
\hline UPV. 12 & .086 & .31 & .45 & .008 & .003 & 8.81 & 1.00 & .10 & .0062 & .08 & .045 & .23 & $.0048 B$ & 12.3 & 3.1 & Si & Trazas \\
\hline UPV. 15 & .10 & .35 & .47 & .020 & .004 & 8.20 & .91 & .26 & .055 & .075 &.. & .24 & - & 11.6 & 4.9 & No & 0 \\
\hline 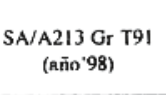 & $\begin{array}{c}.08 \\
+ \\
.12\end{array}$ & $\begin{array}{l}.20 \\
\div \\
.50\end{array}$ & $\begin{array}{l}.30 \\
+ \\
.60\end{array}$ & $\begin{array}{c}s \\
.020\end{array}$ & $\begin{array}{l}\leq \\
.010\end{array}$ & $\begin{array}{c}8.00 \\
\div \\
9.50\end{array}$ & $\begin{array}{c}.85 \\
\div \\
1.05\end{array}$ & $\begin{array}{c}5 \\
.40\end{array}$ & $\begin{array}{c}.030 \\
+ \\
.070\end{array}$ & $\begin{array}{c}.06 \\
\overline{\div} \\
.10\end{array}$ & - & $\begin{array}{c}.18 \\
\div \\
.25\end{array}$ & $\begin{array}{c}\mathrm{Al} \\
\mathrm{S} \\
.04\end{array}$ & - & -. & .. &.. \\
\hline
\end{tabular}

TABLA II. PROPIEDADES MECÁNICAS OBTENIDAS A TRACCIÓN Y TEMPERATURA AMBIENTE DESPUÉS DE NORMALIZAR LOS ACEROS A $1050^{\circ} \mathrm{C}$ (1 HORA) Y REVENIR A $750^{\circ} \mathrm{C}$ (1 HORA). (EN TODOS LOS CASOS SE CUMPLE CON LOS VALORES ESPECIFICADOS POR ASME/ ASTM PARA LA CALIDAD SA/A 213 GR T91)

\begin{tabular}{|c|c|c|c|c|c|c|}
\hline $\begin{array}{l}\text { ACERO } \\
\left(N^{\bullet}\right)\end{array}$ & $\begin{array}{l}\text { COMPOSICION } \\
\text { (de identificacion) }\end{array}$ & $\begin{array}{c}\operatorname{Re}(0.2 \%) \\
\mathrm{N}^{\prime} / \mathbf{m m}^{2} \\
(\mathrm{MPa})\end{array}$ & $\begin{array}{c}\mathbf{R}_{\mathbf{m}} \\
\mathbf{N} / \mathbf{m m}^{2} \\
(\mathbf{M P a})\end{array}$ & $\underset{(\%)}{A}$ & $\begin{array}{c}Z \\
(\%)\end{array}$ & $\mathbf{R e} / \mathbf{R m}$ \\
\hline UPV.1 & Base-Experimental & 590 & 731 & 23 & 74 & 0.81 \\
\hline UPV. 2 & $0.05 \% \mathrm{C}$ & 586 & 707 & 23 & 77 & 0.83 \\
\hline UPV. 3 & $\begin{array}{l}0.03 \% \mathrm{C}, \\
0.07 \% \mathrm{~N}\end{array}$ & 652 & 759 & 21 & 76 & 0.86 \\
\hline UPV. 8 & $0.50 \% \mathrm{~V}$ & 524 & 679 & 26 & 75 & 0.77 \\
\hline UPV.9 & $0.15 \% \mathrm{Nb}$ & 603 & 726 & 20 & 74 & 0.83 \\
\hline UPV.11 & $0.002 \% \mathrm{P}$ & $\$ 92$ & 727 & 23 & 74 & 0.81 \\
\hline UPV.12 & $\begin{array}{l}0.04 \% \mathrm{Ti} \\
0.005 \% \mathrm{~B}, \\
0.006 \% \mathrm{~N}\end{array}$ & 526 & 676 & 22 & 77 & 0.78 \\
\hline UPV.15 & Base-Comercial & 592 & 740 & 24 & 73 & 0.80 \\
\hline \multicolumn{2}{|c|}{$\underset{\text { (แn்' }}{\mathrm{SA} / \mathrm{A} \text { ) } 213 \mathrm{Gr}}$} & 2415 & 2585 & 220 & 240 & 20.71 \\
\hline
\end{tabular}

en caliente a barras de $20 \mathrm{~mm}$ de $\phi$, además una colada de acero comercial (UPV. 15) fue procesada en las mismas condiciones. Acontinuaciónse trataron térmicamente mediante Normalizado a $1050^{\circ} \mathrm{C}(1 \mathrm{~h})$ y Revenido a $750^{\circ} \mathrm{C}(1 \mathrm{~h})$.

La composición y características de las coladas UPV.1 y UPV.15 cumplen con las indicadas por ASME/ASTM para su grado T91/P91, en la composición de las coladas restantes (UPV.2, 3, 8, 9, 11 y 12) se ha variado el contenido de algunos elementos $(\mathrm{C}, \mathrm{N}, \mathrm{V}, \mathrm{Nb}, \mathrm{Ti})$ y en la colada UPV. 12 además se ha adicionado Boro (B). La composición química ha sido obtenida mediante las técnicas analíticas instrumentales de espectrometría de emisión atómica por descarga eléctrica en alto vacío (AES) y por acoplamiento de plasma inductivo (ICP). Tabla I. Las características mecánicas a tracción y temperatura ambiente han sido obtenidas en máquinas universales electromecánicas y sus resultados se muestran en la Tabla II.

\subsection{Desarrollo}

Todos los aceros fueron austenizados a $1050^{\circ} \mathrm{C}(1 \mathrm{~h})$, enfriados al aire en calma y revenidos a $750^{\circ} \mathrm{C}(1 \mathrm{~h})$. El acero de referencia UPV-15, además, fue austenizado y revenido a otras temperaturas, según : $1050 / 730^{\circ} \mathrm{C}, 1050 / 750^{\circ} \mathrm{C}, 1050 / 780^{\circ} \mathrm{C}$, $1100 / 750^{\circ} \mathrm{C}, 1100 / 780^{\circ} \mathrm{C}$ y $1200 / 750^{\circ} \mathrm{C}$. La temperatura usual de servicio de los aceros $9 \mathrm{Cr} 1 \mathrm{MoVNb}$ viene a estar en $600 \pm 50^{\circ} \mathrm{C}$, es por ello, por lo cual las temperaturas que se eligieron para los ensayos de fluencia, fueron de $600-650^{\circ} \mathrm{C}$ y las tensiones (s) aplicadas (de forma constante y continuada) fueron de 124, 139 y $154 \mathrm{~N} / \mathrm{mm}^{2}$, (para $\mathrm{T}=600^{\circ} \mathrm{C}$ ), que se corresponden con el 30, 33.5 y $37 \%$, respectivamente, del límite elástico mínimo (Re mín) especificado por ASME/ASTM y de 62, 77 y $93 \mathrm{~N} / \mathrm{mm}^{2}$ (para $\mathrm{T}=650^{\circ} \mathrm{C}$ ) que, asimismo se corresponden con el $15 \%$, 18.5 y $22.5 \%$ del Re mín, respectivamente.

En los ensayos de fluencia se utilizaron máquinas de ensayo de peso muerto de la firma inglesa Mayes y los resultados obtenidos se muestran en la Tabla III. En la Tabla IV se muestran los resultados del acero comercial UPV-15 el cual también se ensayó a $550^{\circ} \mathrm{C}$ y en la Tabla $\mathrm{V}$ podemos ver los valores que adoptan los parámetros de Larson-Miller (16), Manson-Haferd (17) y de OrrSherby-Dorn (18) para los aceros UPV.3, 8, 12 y 15.

En todos los casos las probetas de ensayo se obtuvieron paralelas a la dirección de laminación y su morfología y dimensiones se tomaron del trabajo de Middleton et al. (19). Figura 1. 
TAbla III. VAlores obtenidos de $\mathbf{t}$ A ROtura y de A/Z PARA Diferentes Y CTES. $\sigma$. PARA $\mathbf{T}=600$ Y $650^{\circ} \mathrm{C}$.

\begin{tabular}{|c|c|c|c|c|c|c|c|c|c|c|c|c|c|c|c|c|c|}
\hline \multirow{3}{*}{$\begin{array}{c}\mathrm{T} \\
\left({ }^{\circ} \mathrm{C}\right)\end{array}$} & \multirow{3}{*}{$\begin{array}{c}\sigma \\
\left(\mathbf{N} / \mathbf{m m}^{2}\right)\end{array}$} & \multicolumn{16}{|c|}{$\begin{array}{c}\mathrm{N}^{0} \text { de acero } \\
\mathrm{TT}=\mathrm{N}\left(1050^{\circ} \mathrm{C}\right)+\mathrm{R}\left(750^{\circ} \mathrm{C}\right)\end{array}$} \\
\hline & & \multicolumn{4}{|c|}{ UPV-3 } & \multicolumn{4}{|c|}{ UPV-8 } & \multicolumn{4}{|c|}{ UPV-12 } & \multicolumn{4}{|c|}{ UPV-15 } \\
\hline & & (h) & L-M & M-H & OSD & (h) & L-M & M-H & OSD & (h) & L-M & M-H & OSD & (h) & L-M & M-H & OSD \\
\hline \multirow{3}{*}{600} & 154 & - & $\cdot$ & $\cdot$ & - & . & $\cdot$ & $\cdot$ & - & - & $\cdot$ & $\cdot$ & $\cdot$ & 1133 & 28,85 & $-33,88$ & $-18,71$ \\
\hline & 139 & - & $\cdot$ & $\cdot$ & $\cdot$ & $\cdot$ & $\cdot$ & - & $\cdot$ & $\cdot$ & $\cdot$ & $\cdot$ & $\cdot$ & $\cdot$ & - & - & $\cdot$ \\
\hline & 124 & . & $\cdot$ & - & - & $\cdot$ & - & - & - & $\cdot$ & $\cdot$ & $\cdot$ & - & 6.308 & 29.51 & .31 .14 & $-17,96$ \\
\hline \multirow{3}{*}{650} & 93 & - & - & $\cdot$ & $\cdot$ & $\cdot$ & $\cdot$ & $\cdot$ & $\cdot$ & 118 & 29,60 & $-31,67$ & -18.52 & 783 & 30,36 & $-29,13$ & $-17,70$ \\
\hline & 77 & 347 & 30,03 & -30.22 & $-18,05$ & 11,87 & 30.32 & $-28,58$ & -17.52 & 334 & 30,02 & -30.28 & -18.07 & 2306 & 310.79 & -27.68 & -17.23 \\
\hline & 62 & 810 & 30,37 & -29.07 & -17.68 & . & - & - & - & & - & - & - & - & . & - & - \\
\hline
\end{tabular}

Tabla IV. Acero UPV. 15. Valores obtenidos de $\mathbf{t}$ A Rotura y de A/Z Para distintos y Ctes. $\sigma . \mathrm{Para} T=600 \pm 50^{\circ} \mathrm{C}$ y $\neq \mathrm{N}+\mathrm{R}$.

\begin{tabular}{|c|c|c|c|c|c|c|c|c|c|c|c|c|c|}
\hline \multirow{3}{*}{$\begin{array}{c}\mathrm{T} \\
\left({ }^{\circ} \mathrm{C}\right)\end{array}$} & \multirow{3}{*}{$\begin{array}{c}\sigma \\
\left(\mathrm{N} / \mathrm{mm}^{2}\right)\end{array}$} & \multicolumn{12}{|c|}{ Normalizado/Revenido } \\
\hline & & \multicolumn{2}{|c|}{$1050 / 730$} & \multicolumn{2}{|c|}{$1050 / 750$} & \multicolumn{2}{|c|}{$1050 / 780$} & \multicolumn{2}{|c|}{$1100 / 750$} & \multicolumn{2}{|c|}{$1100 / 780$} & \multicolumn{2}{|c|}{$1200 / 750$} \\
\hline & & (h) & $\mathbf{A} / \mathbf{Z}$ & (h) & $\mathbf{A} / \mathbf{Z}$ & (b) & $\mathbf{A} / \mathbf{Z}$ & (h) & $\mathbf{A} / \mathbf{Z}$ & (b) & $\mathbf{A} / \mathbf{Z}$ & (h) & $\mathbf{A} / \mathbf{Z}$ \\
\hline \multirow[b]{2}{*}{550} & 216 & - & - & $\underline{6431}$ & $31 / 83$ & - & - & $\cdot$ & - & $\cdot$ & - & - & - \\
\hline & 185 & - & - & $\begin{array}{c}> \\
8680\end{array}$ & - & - & - & - & $\cdot$ & - & - & - & - \\
\hline \multirow{3}{*}{600} & 154 & - & - & 1133 & $35 / 87$ & - & - & - & - & - & - & $\cdot$ & $\cdot$ \\
\hline & 139) & $\begin{array}{c}> \\
2271\end{array}$ & $\cdot$ & - & - & $\underline{1197}$ & $+1 / 89$ & $\begin{array}{c}> \\
4855\end{array}$ & - & $\underline{2271}$ & $34 / 87$ & $\begin{array}{c}> \\
2271\end{array}$ & - \\
\hline & 124 & - & . & 6308 & $36 / 89$ & $\begin{array}{c}> \\
2915\end{array}$ & - & $\begin{array}{c}> \\
405\end{array}$ & - & - & - & $\begin{array}{c}> \\
3285\end{array}$ & - \\
\hline \multirow{3}{*}{650} & 93 & $\cdot$ & - & $\underline{783}$ & $39 / 91$ & - & - & - & - & - & - & $\cdot$ & - \\
\hline & 77 & - & $\cdot$ & $\underline{2306}$ & $48 / 91$ & $\cdot$ & - & - & - & $\cdot$ & $\cdot$ & $\cdot$ & - \\
\hline & 62 & . & - & - & - & . & - & - & - & - & - & . & $\cdot$ \\
\hline
\end{tabular}

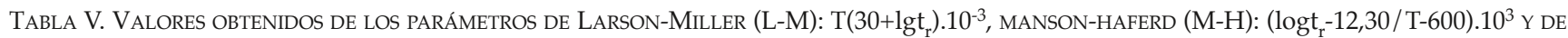
ORR-SHERBY-DORN (OSD): $\log \mathrm{t}_{\mathrm{r}}-19000 / \mathrm{T}$.

\begin{tabular}{|c|c|c|c|c|c|c|c|c|c|c|c|c|c|}
\hline \multirow{3}{*}{$\begin{array}{c}\mathrm{T} \\
\left({ }^{\circ} \mathrm{C}\right)\end{array}$} & \multirow{3}{*}{$\begin{array}{c}\sigma \\
\left(\mathrm{N} / \mathrm{mm}^{2}\right)\end{array}$} & \multicolumn{12}{|c|}{$\begin{array}{c}\mathrm{N}^{\circ} \mathrm{de} \text { acero } \\
{\left[\mathrm{TT}=\mathrm{N}\left(1050^{\circ}\right)+\mathrm{R}\left(750^{\circ} \mathrm{C}\right)\right]}\end{array}$} \\
\hline & & \multirow{2}{*}{$\begin{array}{l}\text { UPV.1 } \\
\text { (h) }\end{array}$} & \multirow{2}{*}{$\begin{array}{c}\text { UPV.2 } \\
\text { (h) }\end{array}$} & \multicolumn{2}{|c|}{ UPV.3 } & \multicolumn{2}{|c|}{ UPV.8 } & \multirow{2}{*}{$\begin{array}{c}\text { UPV.9 } \\
\text { (h) }\end{array}$} & \multirow{2}{*}{$\begin{array}{l}\text { UPV.11 } \\
\text { (b) }\end{array}$} & \multicolumn{2}{|c|}{ UPV.12 } & \multicolumn{2}{|c|}{ UPV.15 } \\
\hline & & & & (h) & $\overline{A Z}$ & (h) & $\bar{A} / \mathbf{Z}$ & & & (h) & $\mathbf{A} / \mathbf{Z}$ & (h) & $\bar{A} / \mathbf{Z}$ \\
\hline \multirow{3}{*}{600} & 154 & $\cdot$ & $>915$ & - & $\cdot$ & $\cdot$ & $\cdot$ & . & $>1117$ & - & - & $\underline{1133}$ & $35 / 87$ \\
\hline & 139 & $>430$ & $\cdot$ & - & $\cdot$ & . & - & $\cdot$ & $>1482$ & - & - & $\cdot$ & - \\
\hline & 124 & $>890$ & - & - & . & $\cdot$ & $\cdot$ & - & - & - & $\cdot$ & 6308 & $36 / 89$ \\
\hline \multirow{3}{*}{6.0} & 93 & - & - & $>117$ & - & $\cdot$ & - & - & $\cdot$ & $\underline{118}$ & $67 / 93$ & $\underline{783}$ & $39 / 91$ \\
\hline & 77 & $>+25$ & $>1001$ & 347 & $66 / 92$ & $\underline{1187}$ & $+3 / 91$ & - & $>1659$ & 334 & $72 / 94$ & 2306 & $+8 / 91$ \\
\hline & 62 & $>1220$ & $>1730$ & $\underline{810}$ & $54 / 90$ & $>1680$ & $\cdot$ & $>1680$ & $>792$ & $\cdot$ & $\cdot$ & $\cdot$ & - \\
\hline
\end{tabular}

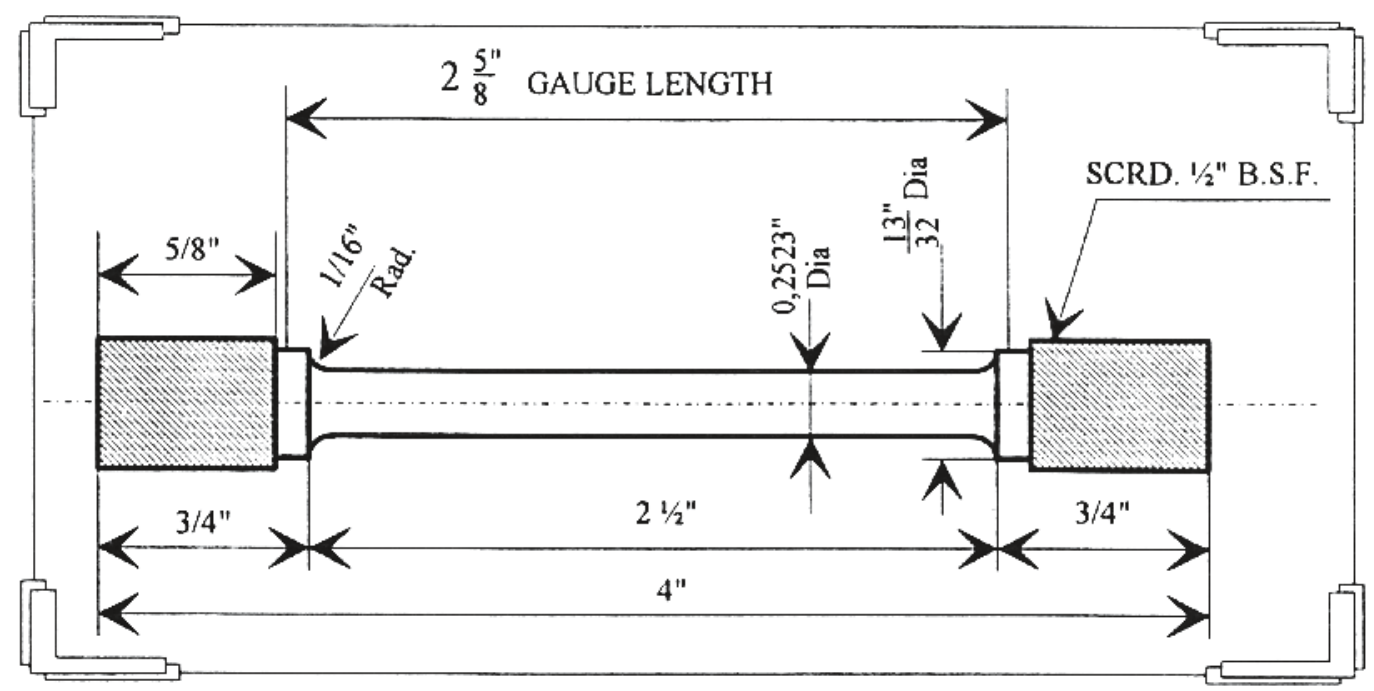

Figura 1. Probeta de ensayo 


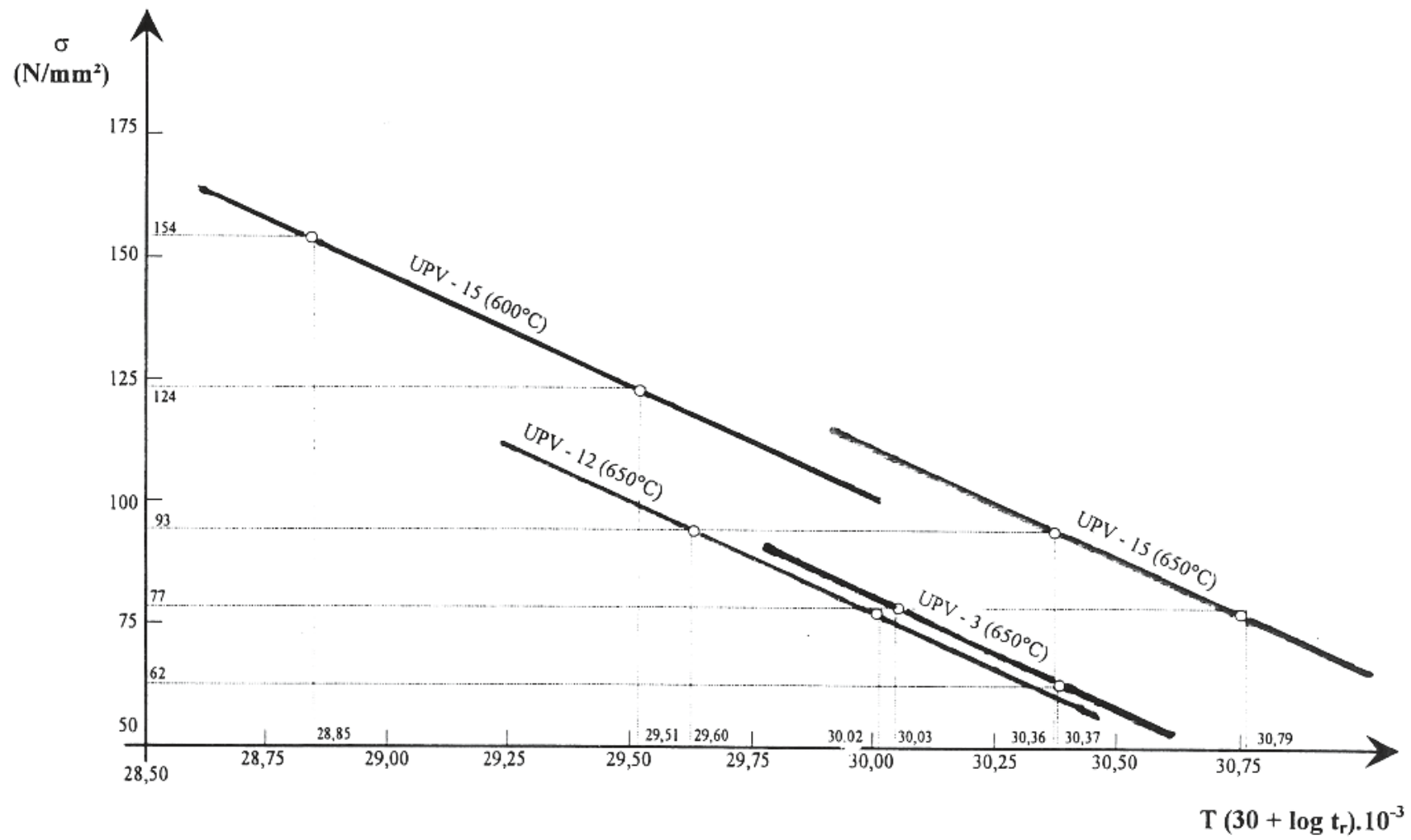

Figura 2. Comportamiento a fluencia. Relación de $\sigma$ con el parámetro de Larson - Miller (L-M).

\section{RESULTADOS Y DISCUSION}

\subsection{Respecto a la relación : composición-comportamiento}

i) El peor comportamiento a fluencia lo presentó el acero UPV. 12. La rotura acaece a las $118 \mathrm{~h}\left(\sigma=93 / \mathrm{mm}^{2}, \mathrm{~T}=650^{\circ} \mathrm{C}\right)$ y $334 \mathrm{~h}\left(\sigma=77 \mathrm{~N} / \mathrm{mm}^{2}, \mathrm{~T}=650^{\circ} \mathrm{C}\right)$, respectivamente. Tabla III. Este es el único acero al cual se le adicionó B (48 ppm) y Ti $(0,045 \%)$. Además es el de más bajo contenido en N (62 ppm). Tabla I. Le sigue el acero UPV.3 en el cual la rotura acaece a las $437 \mathrm{~h}\left(\mathrm{~s}=77 \mathrm{~N} / \mathrm{mm}^{2}, \mathrm{~T}=650^{\circ} \mathrm{C}\right)$ y $810 \mathrm{~h}\left(\mathrm{~s}=62 \mathrm{~N} / \mathrm{mm}^{2}, \mathrm{~T}=\right.$ $\left.650^{\circ} \mathrm{C}\right)$, respectivamente. Tabla III. Este tiene el más bajo contenido en $C(0,031 \%)$ y más alto contenido en $N(730$ ppm). Tabla I.

ii) El mejor comportamiento a fluencia lo presentaron los aceros UPV.1 (experimental) y UPV.15 (comercial), cuyas composiciones cumplen con las especificadas por ASME/ASTM para su grado T91/P91.

iii) El cromo equivalente (Cr eq) de la expresión matemática de Bodine et al. (20) y el factor de ferrita de Kaltenhauser (21) adoptan los valores de $13 \%$ y $10 \%$ respectivamente, para los aceros UPV.12 y 3 (de peor comportamiento a fluencia) y de 10\% y 7\%, asimismo respectivamente, para los aceros de mejor comportamiento a fluencia, cuales son los UPV. 1 y 15.

\subsection{Respecto a la relación : propiedades mecánicas-compor- tamiento}

i) Uno de los aceros de peor comportamiento a fluencia (el UPV.3) presenta su relación Límite Elástico (Re)/Resistencia Mecánica (Rm) más alta : $\operatorname{Re} / \mathrm{Rm}=0,86$

ii) En todos los casos los valores obtenidos de ductilidad a rotura por fluencia han sido altos, variando de $(\mathrm{A} / \mathrm{Z})$ mín = 39/31 hasta $(\mathrm{A} / \mathrm{Z})$ máx $=72 / 94$. Tablas III y IV.

\subsection{Respecto a la relación : estructura-comportamiento}

i) Utilizando los diagramas de Schneider (22) para la predicción de microestructuras (Tabla I) y confirmándose mediante examen metalográfico, observamos que los aceros que tienen mejor comportamiento a fluencia (UPV. 1 y 15) presentan una estructura completamente martensítica y aquellos que tienen un peor comportamiento a fluencia (aceros UPV. 12 y

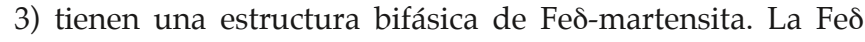
preferentemente se observa en borde de grano.

ii) El acero UPV.15 $\left(1050 / 750^{\circ} \mathrm{C}\right)$ observado al microscopio electrónico de barrido (MEB) mostró abundantes precipitados del tipo $\mathrm{M}_{23} \mathrm{C}_{6} \mathrm{y}$ en menor proporción de $\mathrm{V}_{4} \mathrm{C}_{3}$ y $\mathrm{NbC}$.

\subsection{Respecto al comportamiento a fluencia}

i) El mejor comportamiento a fluencia lo presentan los aceros UPV.1 y 15 y el peor los aceros UPV.12 y 3. Figuras 2 y 3. Entendemos que el peor comportamiento de éstos últimos aceros se debe a que en ellos se cumple lo siguiente :

${ }^{*}$ Cr eq (de Bodine (20)) $>12 \%$

${ }^{*}$ FFK (de Kaltenhauser (21)) $>8 \%$

* $\mathrm{C}+\mathrm{N}<0,14 \%$ (en este caso son bastante bajos, $\mathrm{C}+\mathrm{N}=$ $0.09 \div 0,1 \%)$

* Presentan Feঠ retenida y por ende estructura bifásica de Fed-martensita.

ii) Asimismo entendemos que el peor comportamieto a fluencia del acero UPV.12 se debe a que es el único acero que contiene B (48 ppm) y Ti $(0,045 \%)$. El B con su contribución a formar fases secundarias y promocionar la formación de Fed en borde de grano y el $\mathrm{Ti}$ con su tendencia a formar compuestos de Ti $(\mathrm{C}, \mathrm{N})$ hacen aquel más susceptible.

Observaciones que están de acuerdo con la bibliografía especializada al respecto $(23,24,25)$. 


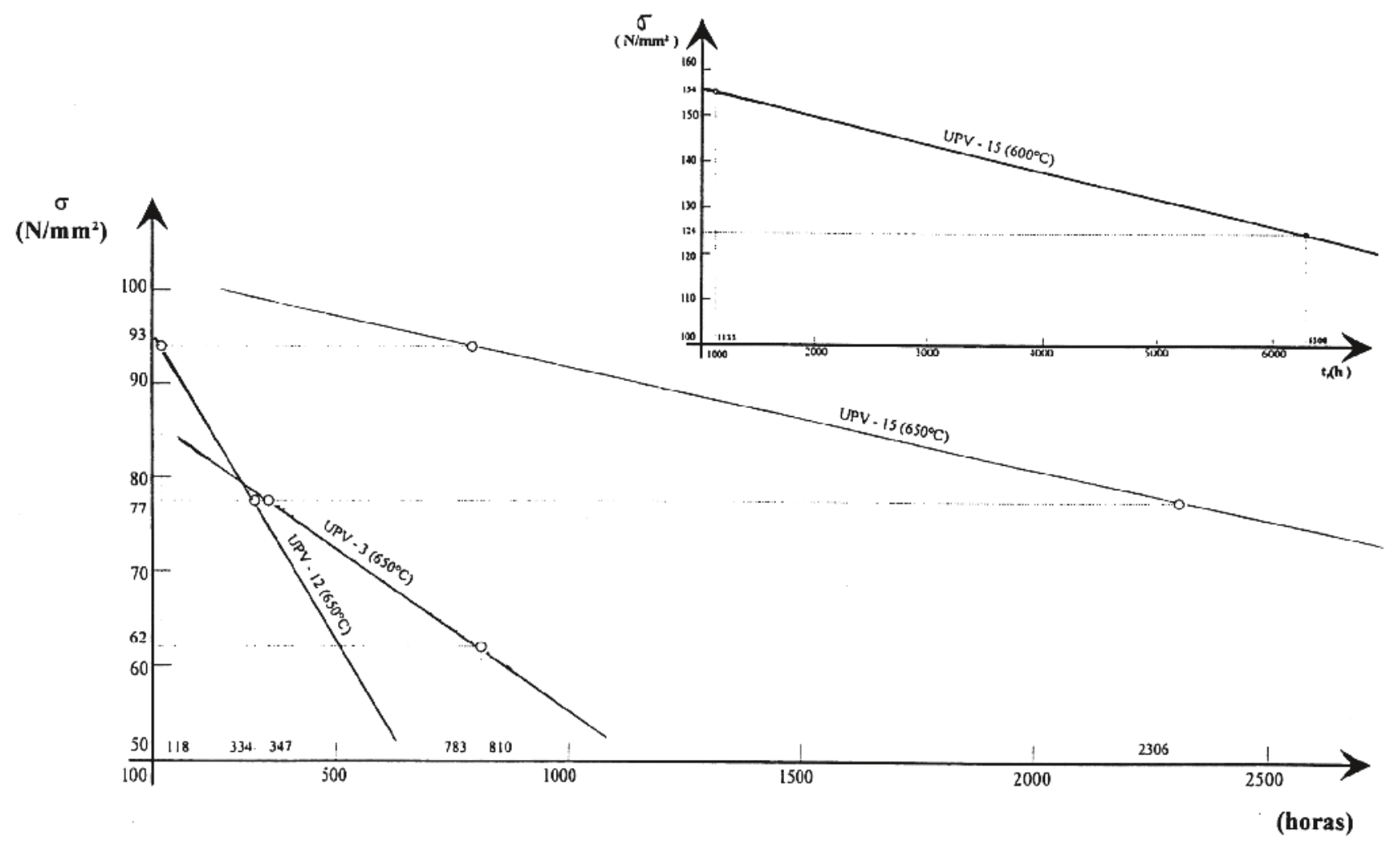

Figura 3. Comportamiento a fluencia. Relación de $\sigma$ con el $\mathrm{t}_{\mathrm{r} .}\left(\mathrm{T}=600\right.$ y $\left.650^{\circ} \mathrm{C}\right)$.

\section{CONCLUSIONES}

Del presente trabajo experimental se pueden sacar las principales conclusiones :

i) Se confirma el buen comportamiento a fluencia de los aceros del tipo $9 \mathrm{Cr} 1 \mathrm{MoVNb}$ cuando su composición y características cumplen con las especificadas en norma (ASTM) y código de diseño ASME. Independientemente de si el acero es fabricado y procesado en Laboratorio o por medios industriales. Una buena ductilidad a rotura es asimismo observada.

ii) Modificar la composición química standard del acero tipo $9 \mathrm{CrMoVNb}$, adicionando Ti a ordenes del 0,05\% (para refinar el grano) y B a ordenes de 50 ppm (para aumentar la templabilidad) decrecen la resistecia a fluencia.

iii) La estructura completamente martensítica tiene mejor comportamiento a fluencia que la estructura bifásica de Fedmartensita. La fractura se inicia en la Fed.

\section{BIBLIOGRAFIA}

1. K. Bell. “Report n 598/1997 (Confidential). Ref. 7061. 02. 96 / 723. 03”. TWI. Abington. Cambridge. UK.March. 1997.

2. ASME. Section II: Part. A "Specification for Seamless Ferritic and Austenitic Alloy-Steel Boiler, Superheater, and Heat-Exchanger Tubes". SA 213/SA 2123M. 271-280. New York. USA. July 1995.

3. ASTM. Section 1. Volume 01.01. "Standard Specification for Seamless Ferritic and Austenitic Alloy Steel Boiler, Superheater, and Heat-Exchanger Tubes". A213/ A213M. 111-117. Philadelphia. USA. (1991).

4. ASME. Section II. Part. A. "Specification for Seamless Ferritic Alloy-Stell Pipe for High-Temperature Service". SA 335/335M. 463-474. New York. USA. July. 1995.

5. E.L. Bergquist. "Consumables and Welding Modified 9Cr1Mo Steel. Svetsaren No. 1 (22-25). Göteborg. Sweden. 1999.

6. P.F. Aplin and C.J. Middleton. "The Development of High Strength $9 \mathrm{Cr}$ Steels for Steam based Power Plant for the 1990's": Proc. Conf. : Steam Plant for the 90's. 1MechiE. 140-160. London. 1990.
7. E. Metcalfe et al. "CECB Development Programmes on Ferritic Materials for Advanced Plants". Proc. 2d Int. Conf. : Improved Coal Fired Power Plants. 19.1 (19-28). EPRI. Palo Alto. USA. 1998.

8. V.K. Sikka et. At. "Creep Properties of Modified 9Cr1Mo Steel". ORNL. 6299. USA. September. 1996.

9. S. Gutierrez et al. "Life Optimization of Dissimilar Metal Welds for High Temperature Design : P22, P91 and Esshete 1250". Proyecto Brite-Euram n ${ }^{\circ}$ BE-7301 (BRE.CT.94.0939). 1997.

10. T. Kanero et al. "Modified 9Cr Steel Boiler Tube and Pipe". NKK. Confidential Report. No 105 (226-233). July (1998).

11. A.M. Barnes. "Report n ${ }^{\circ}$ 534/1995 (Confidential): Ref 7224.02/95/885.3". TWI. Abington. Cambridge. UK. December. 1995.

12. J.F. Arav and J.C. Van Wortel. "Properties and Application of the Modified 9Cr Steel T91/P91". Stainless Steel. Europe. April. 1995.

13. F. Arav and J.C. Van Wortel. "Investigations into the Properties of Modified 9Cr Steel for High Temperatures Applications". TNO. Cofidential Report No T91 (30-94). Holanda. 1999.

14. S. Gutierrez, J.M. San Juan y O. Ruano. "Estudio de la influencia de la variación en los contenidos de $\mathrm{C}, \mathrm{N}, \mathrm{V}, \mathrm{Nb}, \mathrm{B}, \mathrm{Cr}$ y W sobre las propiedades mecánicas de los aceros del tipo $9 \mathrm{Cr} 1 \mathrm{MoVb}$ utilizados para servicios a altas temperaturas": Libro de Actas. Editor. X. Gil Mur. 284-301. ETSII. Barcelona. 1996.

15. S. Gutierrez, J.M. San Juan y A. Valea "Estudio de la influencia del incremento de la temperatura de austenización sobre el crecimiento de grano en aceros ferrítico-martensíticos del tipo $9 \mathrm{Cr} 1 \mathrm{MoVNb}$ utilizados en elementos presurizados para altas T de servicio". Libro de Actas. Editores : M. Carsí et al. 297304. Madrid. 1998.

16. F.R. Larson and J. Miller. "Trans. ASME". Vol 74 (765). (1952).

17. S.S. Manson ad A.M. Haferd. "A linear Time-Temperature relation for Extrapolation of Creep and Stress Rupture Data". NASA. No TN 2890. March. 1952.

18. R.L. Orr, O.D. Sherby and J.E. Dorn. “Trans. ASME”. Vol 46 (113). 1954.

19. C.C. Middleton et al. "A review of Laboratory data in High Chromium Ferritic Steels". ImechE (275-282). London 1990.

20. G.C. Bodine. "A program for the Development of Advanced Ferritic Alloys for LMFBR Structural Applications". ORL. Ref. Sub 4291/1. USA. 1977.

21. R.H. Kaltenhauser. "Improving the Engineering Properties of Ferritic Stainless Steels". Met. Eng. Quart. Vol 11 (2). 41-47. May (1971).

22. H. Schneider. "Investment Casting of High Strength $12 \mathrm{Cr}$ Steel". Foondry Trade J. Vol 108 (5) (1960). 
23. M.F. Ashby and B.F. Dyson. "Creep Damage Mechanics and Micromechanisms". Proc. of ICF6. Vol 1 (1994).

24. R. Viswanathan. "Damage Mechanisms and Life Assessment of High Temperature Components". ASM. USA. 1998.
25. V. Von Estorff and H. Stamm. "Deformation of Creep Damage in Steels". Commision of the European Communities. Inst. of Advanced Materials. ISPRA. Proc. Fifth Int. Conf. on Creep of Materials. (287-296). Florida. 1992. 